


\title{
Continuous Time Dynamical Systems
}

\section{State Estimation and Optimal Control with Orthogonal Functions}

\author{
B. M. Mohan \\ S. K. Kar
}

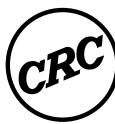




\title{
CRC Press
}

Taylor \& Francis Group

6000 Broken Sound Parkway NW, Suite 300

Boca Raton, FL 33487-2742

(C) 2013 by Taylor \& Francis Group, LLC

CRC Press is an imprint of Taylor \& Francis Group, an Informa business

No claim to original U.S. Government works

Printed in the United States of America on acid-free paper

Version Date: 20120822

International Standard Book Number: 978-1-4665-1729-5 (Hardback)

This book contains information obtained from authentic and highly regarded sources. Reasonable efforts have been made to publish reliable data and information, but the author and publisher cannot assume responsibility for the validity of all materials or the consequences of their use. The authors and publishers have attempted to trace the copyright holders of all material reproduced in this publication and apologize to copyright holders if permission to publish in this form has not been obtained. If any copyright material has not been acknowledged please write and let us know so we may rectify in any future reprint.

Except as permitted under U.S. Copyright Law, no part of this book may be reprinted, reproduced, transmitted, or utilized in any form by any electronic, mechanical, or other means, now known or hereafter invented, including photocopying, microfilming, and recording, or in any information storage or retrieval system, without written permission from the publishers.

For permission to photocopy or use material electronically from this work, please access www.copyright. com (http://www.copyright.com/) or contact the Copyright Clearance Center, Inc. (CCC), 222 Rosewood Drive, Danvers, MA 01923, 978-750-8400. CCC is a not-for-profit organization that provides licenses and registration for a variety of users. For organizations that have been granted a photocopy license by the CCC, a separate system of payment has been arranged.

Trademark Notice: Product or corporate names may be trademarks or registered trademarks, and are used only for identification and explanation without intent to infringe.

\section{Library of Congress Cataloging-in-Publication Data}

\author{
Mohan, B. M. (Bosukonda Murali) \\ Continuous time dynamical systems : state estimation and optimal control with \\ orthogonal functions / B.M. Mohan, S.K. Kar. \\ p. $\mathrm{cm}$. \\ Includes bibliographical references and index. \\ ISBN 978-1-4665-1729-5 (hardback) \\ 1. Functions, Orthogonal. 2. Differentiable dynamical systems--Automatic \\ control--Mathematics. 3. Mathematical optimization. I. Kar, S. K. II. Title.
}

QA404.5.M64 2012

$515.55--\mathrm{dc} 23$ 


\title{
Dedication
}

\author{
To my wife, Vijaya Lakshmi, \\ my son, Divyamsh \& \\ my daughter, Tejaswini
}

B. M. Mohan

To my parents, Satya Ranjan \& Manjushree,

$$
\begin{aligned}
& \text { my sister, Sanjeeta, } \\
& \text { my wife, Swastika \& }
\end{aligned}
$$

my daughters, Santika \& the late Ayushi

S. K. Kar 
$\because$ Taylor \& Francis

Taylor \& Francis Group

http://taylorandfrancis.com 


\section{Contents}

List of Abbreviations

List of Figures

Preface

xix

Acknowledgements

xxi

About the Authors

xxiii

1 Introduction

1.1 Optimal Control Problem . . . . . . . . . . . 1

1.2 Historical Perspective . . . . . . . . . . . . 3

1.3 Organisation of the Book . . . . . . . . 8

2 Orthogonal Functions and Their Properties 11

2.1 Introduction . . . . . . . . . . . . 11

2.2 Block-Pulse Functions (BPFs) . . . . . . . . 14

2.2.1 Integration of $\boldsymbol{B}(t) \ldots \ldots \ldots$

2.2.2 Product of two BPFs . . . . . . . . 16 
2.2.3 Representation of $C(t) \mathbf{f}(t)$ in terms of BPFs 16

2.2.4 Representation of a time-delay vector in BPFs ............... 17

2.2.5 Representation of reverse time function vector in BPFs . . . . . . . . . . . 18

2.3 Legendre Polynomials (LPs) . . . . . . . . . . . . 19

2.4 Shifted Legendre Polynomials (SLPs) . . . . . . . . 20

2.4.1 Integration of $\boldsymbol{L}(t) \quad \ldots \ldots \ldots$

2.4.2 Product of two SLPs . . . . . . . . . . . . 23

2.4.3 Representation of $C(t) \mathbf{f}(t)$ in terms of SLPs 24

2.4.4 Representation of a time-delay vector function in SLPs . . . . . . . . . . . 25

2.4.5 Derivation of a time-advanced matrix of SLPs . . . . . . . . . . . . . 27

2.4.6 Algorithm for evaluating the integral in Eq. (2.75) . . . . . . . . . . . . . . 28

2.4.7 Representation of a reverse time function vector in SLPs . . . . . . . . . . 30

2.5 Nonlinear Operational Matrix . . . . . . . . . 30

2.6 Rationale for Choosing BPFs and SLPs . . . . . . . 32

3 State Estimation 35

3.1 Introduction . . . . . . . . . . . . . 35

3.2 Inherent Filtering Property of OFs . . . . . . . 38

3.3 State Estimation . . . . . . . . . . . . . . 39

3.3.1 Kronecker product method . . . . . . . . . 43

3.3.2 Recursive algorithm via BPFs . . . . . . . 43 
3.3.3 Recursive algorithm via SLPs . . . . . . . . 44

3.3.4 Modification of the recursive algorithm of Sinha and Qi-Jie ......... . . . 45

3.4 Illustrative Examples . . . . . . . . . . . . . 47

3.5 Conclusion . . . . . . . . . . . . . . . 58

4 Linear Optimal Control Systems Incorporating Observers

4.1 Introduction . . . . . . . . . . . . . . 65

4.2 Analysis of Linear Optimal Control Systems Incorporating Observers . . . . . . . . . . . . . . 68

4.2.1 Kronecker product method . . . . . . . . 69

4.2.2 Recursive algorithm via BPFs . . . . . . . 70

4.2.3 Recursive algorithm via SLPs . . . . . . . 71

4.3 Illustrative Example . . . . . . . . . . . . . . 72

4.4 Conclusion . . . . . . . . . . . . . . . 77

5 Optimal Control of Systems Described by IntegroDifferential Equations

5.1 Introduction . . . . . . . . . . . . . 79

5.2 Optimal Control of LTI Systems Described by Integro-Differential Equations . . . . . . . . . . 80

5.3 Illustrative Example . . . . . . . . . . . . . . . 84

5.4 Conclusion . . . . . . . . . . . . . 86

6 Linear-Quadratic-Gaussian Control 89

6.1 Introduction . . . . . . . . . . . . . . . 89 
viii

6.2 LQG Control Problem . . . . . . . . . . . . . . . 90

6.3 Unified Approach . . . . . . . . . . . . . . 93

6.3.1 Illustrative example . . . . . . . . . . . 96

6.4 Recursive Algorithms . . . . . . . . . . . . . . 97

6.4.1 Recursive algorithm via BPFs . . . . . . . . 101

6.4.2 Recursive algorithm via SLPs . . . . . . . . 102

6.4.3 Illustrative example . . . . . . . . . . . . 104

6.5 Conclusion . . . . . . . . . . . . . 105

7 Optimal Control of Singular Systems 109

7.1 Introduction . . . . . . . . . . . . . . 109

7.2 Recursive Algorithms . . . . . . . . . . . . . 111

7.2.1 Recursive algorithm via BPFs . . . . . . . 113

7.2.2 Recursive algorithm via SLPs . . . . . . . . 114

7.3 Unified Approach . . . . . . . . . . . . . 115

7.4 Illustrative Examples . . . . . . . . . . . . . . 117

7.5 Conclusion . . . . . . . . . . . . . . . . 121

8 Optimal Control of Time-Delay Systems 125

8.1 Introduction . . . . . . . . . . . . . 126

8.2 Optimal Control of Multi-Delay Systems . . . . . 129

8.2.1 Using BPFs . . . . . . . . . . . . . . . 133

8.2 .2 Using SLPs . . . . . . . . . . . . . . . 135

8.2.3 Time-invariant systems . . . . . . . . . . 137

8.2.4 Delay free systems . . . . . . . . . . . 138 
8.2.5 Illustrative examples . . . . . . . . . . . . 138

8.3 Optimal Control of Delay Systems with Reverse Time Terms . . . . . . . . . . . . . . 146

8.3.1 Using BPFs . . . . . . . . . . . 151

8.3.2 Using SLPs . . . . . . . . . . . . . 153

8.3.3 Illustrative example . . . . . . . . . . . 154

8.4 Conclusion . . . . . . . . . . . . . . . . 155

9 Optimal Control of Nonlinear Systems 159

9.1 Introduction . . . . . . . . . . . . . . . . 159

9.2 Computation of the Optimal Control Law . . . . . 160

9.3 Illustrative Examples . . . . . . . . . . . . 162

9.4 Conclusion . . . . . . . . . . . . . 166

10 Hierarchical Control of Linear Systems 169

10.1 Introduction . . . . . . . . . . . . . 169

10.2 Hierarchical Control of LTI Systems with Quadratic

Cost Functions . . . . . . . . . . . . . . . . 170

10.2.1 Partial feedback control . . . . . . . . . . 172

10.2.2 Interaction prediction approach . . . . . . 173

10.3 Solution of Hierarchical Control Problem via BPFs 174

10.3.1 State transition matrix . . . . . . . 175

10.3.2 Riccati matrix and open-loop compensation vector ................. 177

10.3.3 State vector . . . . . . . . . . . 178

10.3.4 Adjoint vector and local control . . . . . . 180 
10.3.5 Coordination . . . . . . . . . 180

10.3 .6 Error . . . . . . . . . . . . . . 180

10.4 Extension to Linear Time-Varying Systems . . . . . 180

10.5 Computational Algorithm . . . . . . . . . . . 183

10.6 Illustrative Examples . . . . . . . . . . . . . . 184

10.7 Conclusion . . . . . . . . . . . . . . . 190

$\begin{array}{ll}11 \text { Epilogue } & 199\end{array}$

$\begin{array}{ll}\text { Bibliography } & 203\end{array}$

$\begin{array}{ll}\text { Index } & 217\end{array}$ 


\section{List of Abbreviations}

BPFs Block pulse functions

CP1s Chebyshev polynomials of the first kind

CP2s Chebyshev polynomials of the second kind

GOPs General orthogonal polynomials

HFs Haar functions

HPs Hermite polynomials

ISE Integral square error

JPs Jacobi polynomials

LaPs Laguerre polynomials

LPs Legendre polynomials

LQE Linear-quadratic-estimator

LQG Linear-quadratic-Gaussian

LQR Linear-quadratic-regulator

MIMO Multi-input multi-output

NSR Noise-to-signal ratio

OFs Orthogonal functions

SCFs Sine-cosine functions

SCP1s Shifted Chebyshev polynomials of the first kind

SCP2s Shifted Chebyshev polynomials of the second kind 
xii

SJPs Shifted Jacobi polynomials

SLPs Shifted Legendre polynomials

TPBV Two-point boundary-value

WFs Walsh functions 


\section{List of Figures}

2.1 A set of four BPFs. . . . . . . . . . . 15

2.2 A set of four SLPs. . . . . . . . . . . . 21

3.1 Schematic diagram to study the inherent filtering property of OFs. . . . . . . . . . . . . 39

3.2 Filtering property of SLPs. . . . . . . . . 40

3.3 Residual error in signal reconstruction by SLPs. . 41

3.4 Estimate of state-1 with known initial states. . . . 49

3.5 Estimate of state-2 with known initial states. . . . 50

3.6 Estimate of state-1. . . . . . . . . . . 51

3.7 Estimate of state-2. . . . . . . . . . . 52

3.8 Estimate of state-1 with NSR $=0.1 \ldots \ldots 53$

3.9 Estimate of state-2 with $\mathrm{NSR}=0.1 \ldots \ldots 54$

3.10 Estimate of state-1 with known initial states. . . . 55 
3.11 Estimate of state-2 with known initial states. . . . 56

3.12 Estimate of state-3 with known initial states. . . . 57

3.13 Estimate of state-1. . . . . . . . . . . . 58

3.14 Estimate of state-2. . . . . . . . . . . . 59

3.15 Estimate of state-3. . . . . . . . . . . . 60

3.16 Estimate of state- 1 with $\mathrm{NSR}=0.1 \ldots \ldots 1$

3.17 Estimate of state-2 with NSR $=0.1 \ldots \ldots 2$

3.18 Estimate of state-3 with NSR $=0.1 \ldots \ldots 3$

$4.1 \quad$ Error. . . . . . . . . . . . . . . 74

4.2 State variables. . . . . . . . . . . . . 75

4.3 Optimal control law. . . . . . . . . . . 76

5.1 BPF and SLP solutions of control variable. . . . 85

5.2 BPF and SLP solutions of state variable. . . . . 86

6.1 Optimum linear combined estimation and control. . . . . . . . . . . . . . . . . . 93

6.2 Actual, SLP and BPF solutions of $P_{1}(t) \ldots \ldots 9$

6.3 Actual, SLP and BPF solutions of $P_{2}(t) . \quad \ldots . .99$

6.4 Actual, SLP and BPF solutions of $P_{1}(t) . \ldots 104$ 
6.5 Actual, SLP and BPF solutions of $P_{2}(t) \ldots 105$

6.6 Error in $P_{1}(t)$ : recursive SLPs and non-recursive SLPs $[35] . \ldots \ldots \ldots \ldots$

6.7 Error in $P_{2}(t)$ : recursive SLPs and non-recursive

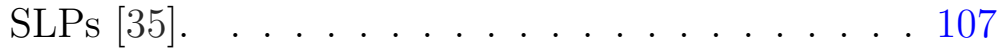

7.1 Actual, SLP and BPF solutions of $u(t) \ldots 119$

7.2 Actual, SLP and BPF solutions of $\mathbf{x}(t) \ldots . . .120$

7.3 SLP and BPF solutions of $\mathbf{u}(t) \ldots \ldots 121$

7.4 SLP and BPF solutions of $\mathrm{x}(t) \ldots \ldots 122$

8.1 Exact, BPF and SLP solutions of $u(t)$ and $x(t)$ variables. . . . . . . . . . . . . . . 139

8.2 BPF, SLP and hybrid function solutions of $u(t)$ and $x(t) \ldots \ldots \ldots \ldots \ldots$

8.3 BPF and SLP solutions of $u(t)$ and $\mathbf{x}(t)$ variables. 142

8.4 BPF, SLP and general Legendre wavelets solutions of $u(t) \ldots \ldots \ldots$. . . . . . . . . . . . . . 144

8.5 BPF, SLP and general Legendre wavelets solutions of $\mathrm{x}(t) \ldots \ldots \ldots \ldots \ldots$

8.6 BPF and SLP solutions of $u(t) \ldots \ldots 147$

8.7 BPF and SLP solutions of $\mathbf{x}(t) \ldots . . . . .148$

8.8 BPF, SLP and hybrid functions solutions of $u_{1}(t) .156$ 
8.9 BPF, SLP and hybrid functions solutions of $u_{2}(t) .157$

8.10 BPF, SLP and hybrid functions solutions of state variables. . . . . . . . . . . . . . . . . 158

9.1 Control variable in Example 1. . . . . . . . . . 163

9.2 State variable in Example 1. . . . . . . . . . . 164

9.3 Control variable in Example 2. . . . . . . . 165

9.4 State variable in Example 2. . . . . . . . . . 166

9.5 Control variable in Example 3. . . . . . . . . . 167

9.6 State variables in Example 3. . . . . . . . . . . 168

10.1 Hierarchical control strategy for a large scale system. . . . . . . . . . . . . . . . 171

10.2 Control variables $u_{1}$ and $u_{2}$. . . . . . . . . 185

10.3 State variables $x_{1}, x_{2}, x_{3}$ and $x_{4} \ldots \ldots$. . 186

10.4 Interaction error behaviour. . . . . . . . . . . . . 187

10.5 A large scale system of four systems and its hierarchical control. . . . . . . . . . . . 187

10.6 Control variables $u_{1}$ and $u_{2}$. . . . . . . . . . 189

10.7 State variables $x_{1}, x_{2}$ and $x_{3} \ldots \ldots \ldots$

10.8 State variables $x_{4}, x_{5}$ and $x_{6} \ldots \ldots 191$ 
10.9 State variables $x_{7}, x_{8}$ and $x_{9} \ldots \ldots 192$

10.10 State variables $x_{10}, x_{11}$ and $x_{12} \ldots \ldots 193$

10.11 Interaction error behaviour. . . . . . . . . . . 194

10.12 Control variables $u_{1}$ and $u_{2}$. . . . . . . . . 195

10.13 State variables $x_{1}$ and $x_{2} \ldots \ldots \ldots 6$

10.14 Interaction error behaviour. . . . . . . . . . 197 
$\because$ Taylor \& Francis

Taylor \& Francis Group

http://taylorandfrancis.com 


\section{Preface}

Orthogonal functions can be broadly classified into three families: the piecewise constant family, the polynomial family, and the sine-cosine family. Each family is classified into different classes of basis functions. Classes such as block-pulse functions, Haar functions and Walsh functions belong to the piecewise constant family while Chebyshev polynomials of the first kind and the second kind, Legendre polynomials, Laguerre polynomials, Hermite polynomials etc. belong to the polynomial family. The techniques of reducing the (differential, integral) calculus of continuous-time dynamical systems to an attractive algebra, approximate in the sense of least squares, emerged in the early 1970s, first with piecewise constant family functions. In subsequent years the use of polynomial family and sine-cosine functions has been demonstrated.

It all began with the Walsh functions application to optimal control problems in 1975, and the block-pulse functions application to state estimation problems in 1984. Since then, blockpulse functions, Legendre polynomials and other classes of basis functions have been applied extensively for state estimation of linear time-invariant systems, and optimal control of varieties of systems such as linear/bilinear/nonlinear, time-invariant/timevarying, and delay-free/delay systems. All these studies show that block-pulse functions and Legendre polynomials have definite computational advantages in comparison to other classes of basis functions, as they can easily lead to recursive algorithms.

The work of the authors and the several interesting contributions of other researchers form the core of this book. It is perhaps the first book devoted exclusively to the application of block-pulse 
$\mathrm{XX}$

functions and Legendre polynomials to state estimation and optimal control of continuous-time dynamical systems. It is hoped that this book will be of interest to control students and engineers.

B. M. Mohan

S. K. Kar 


\section{Acknowledgements}

The authors thank the authorities of the Indian Institute of Technology, Kharagpur and the Institute of Technical Education Research, Sikha 'O' Anusandhan University, Bhubaneswar for the facilities and the conducive atmosphere provided for the research and preparation of this book. They would like to express their gratitude to Professor G. P. Rao and Professor K. B. Datta, both retired from IIT Kharagpur, for their helpful discussions, keen interest and kind encouragement. They are very much indebted to their family members for their love, understanding and support during the years of their research and the period of preparation of this book. Last but not the least, they also thank Dr. Gagandeep Singh, Commissioning Editor, Kari Budyk, Senior Project Coordinator and Karen Simon, Project Editor, of CRC Press, Taylor \& Francis Group, for their continued perseverance and encouragement for making this book a reality. 
$\because$ Taylor \& Francis

Taylor \& Francis Group

http://taylorandfrancis.com 


\section{About the Authors}

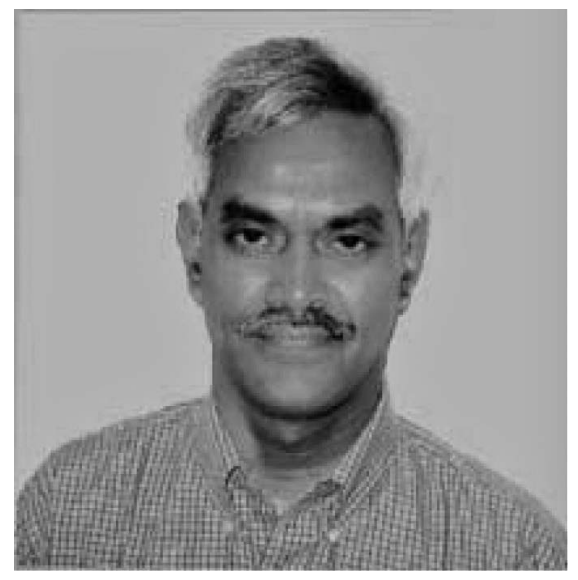

B. M. Mohan received his bachelor's degree in electrical engineering from Osmania University in 1982, a master's degree in electrical engineering (with control systems specialization) from Andhra University in 1985, and his doctoral degree in engineering from the Indian Institute of Technology, Kharagpur in 1989. From July 1989 to October 1990 he was on the faculty of the Electrical and Electronics Engineering Department, Regional Engineering College (now called the National Institute of Technology), Tiruchirapalli. Since November 1990 he has been on the faculty of the Electrical Engineering Department, Indian Institute of Technology, Kharagpur, where he is currently a professor. He was a visiting professor in the Department of Electrical EngineeringSystems, the University of Southern California, Los Angeles, California in 2006.

Dr. Mohan's research interests include identification, analysis, and control of dynamical systems using fuzzy logic and orthogonal functions. He co-authored the research monograph Orthogonal 
xxiv

Functions in Systems and Control (World Scientific, Singapore, 1995), and several papers in international journals and conferences. He is a member of the Asian Control Association, a life member of the Systems Society of India, a senior member of the IEEE, and a life fellow of the Institution of Engineers (India). He is the associate editor of the International Journal of Automation $\&$ Control (IJAAC); an editorial board member of the International Journal of Mathematics and Engineering with Computers (IJMAEC), the Journal of Control Engineering \& Technology (JCET), the International Journal of Engineering Science, Advanced Computing \& Bio-Technology (IJESACBT), and the International Journal of Control, Automation 85 Systems (IJCAS). He is also the editor of the Journal of Electrical \& Control Engineering (JECE), and Current Trends in Systems \& Control Engineering (CTSCE). He was the chair of the Control, Robotics \& Motion Control track, ICIIS 2008 .

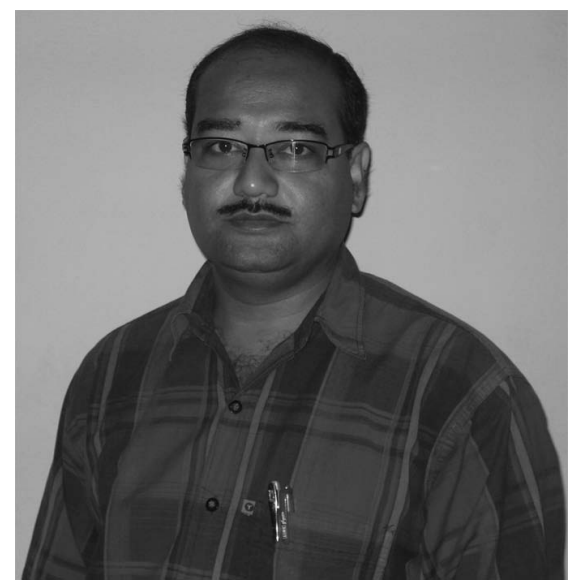

Sanjeeb Kumar Kar received his B.Tech in electrical engineering from the College of Engineering \& Technology (CET), 
OUAT, Bhubaneswar in 1993, and his M. Tech in control systems engineering and Ph.D from the Indian Institute of Technology, Kharagpur, in 2004 and 2011, respectively. From March 2004 to February 2005 he worked as an apprentice engineer on the Odisha State Electricity Board, and from April 1995 to March 1997 as assistant manager in Pal Textile, Balasore. In August 1997 he joined the faculty in electrical engineering, CET, OUAT, Bhubaneswar and continued there until October 2008. Since November 2008, he has been on the faculty of the Electrical Engineering Department, the Institute of Technical Education \& Research (ITER), and Siksha 'O' Anusandhan (SOA) University, Bhubaneswar, where he is currently working as an associate professor and head of the department. 
$\because$ Taylor \& Francis

Taylor \& Francis Group

http://taylorandfrancis.com 


\section{Chapter 1}

\section{Introduction}

The optimal control problem is introduced first. Then optimal control problems of varieties of systems and their solution via different classes of orthogonal functions (OFs) are discussed in the literature review section. The objectives and contributions of the book are stated. The organization of the book is given in the last section.

\subsection{Optimal Control Problem}

A particular type of system design problem is the problem of "controlling" a system. The translation of control system design objectives into the mathematical language gives rise to the control problem. The essential elements of the control problem are

- A desired output of the system.

- A set of admissible inputs or "controls."

- A performance or cost functional which measures the effectiveness of a given "control action."

The objective of the system is often translated into a requirement on the output. Since "control" signals in physical systems are 
usually obtained from equipment which can provide only a limited amount of force or energy, constraints are imposed upon the inputs to the system. These constraints lead to a set of admissible inputs.

Frequently the desired objectives can be attained by many admissible inputs, so the engineer seeks a measure of performance or cost of control which will allow him/her to choose the "best" input. The choice of a mathematical performance functional is a subjective matter. Moreover, the cost functional will depend upon the desired behaviour of the system. Most of the time, the cost functional chosen will depend upon the input and the pertinent system variables. When a cost functional has been decided upon, the engineer formulates his/her control problem as follows:

Determine the admissible inputs which generate the desired output and which optimize the chosen performance measure.

At this point, optimal control theory enters the picture to aid the engineer in finding a solution to his/her control problem. Such a solution, when it exists, is called an optimal control. Optimal control deals with the problem of finding a control law for a given system such that a certain optimality criterion is achieved. An optimal control is a set of differential equations describing the paths of the control variables that minimize the cost functional. The theory of optimal control is concerned with operating a dynamic system at minimum cost. The case where the system dynamics are described by a set of linear differential equations and the cost is described by a quadratic functional is called a linear quadratic problem. One of the main results in the theory is that the solution is provided by the linear-quadratic-regulator (LQR).

In this book different classes of systems are considered with quadratic performance criteria and an attempt is made to find the 
optimal control law for each class of systems using OFs that can optimize the given performance criteria.

\subsection{Historical Perspective}

The Legendre polynomials (LPs) originated from determining the force of attraction exerted by solids of revolution [5], and their orthogonal properties were established by Adrian Marie Legendre during 1784-1790. There is another family of OFs known as piecewise constant basis functions whose functional values are constant within any subinterval of time period. There is a class of complete OFs known as block-pulse functions (BPFs) which is more popular and elegant in the areas of parameter estimation, analysis and control.

Control of linear systems by minimizing a quadratic performance index gives rise to a time-varying gain for the linear state feedback, and this gain is obtained by solving a matrix Riccati differential equation [3]. Probably, Chen and Hsiao, 1975, were the first who applied a class of piecewise constant OFs, i.e. Walsh functions (WFs), obtained a numerical solution of the matrix Riccati equation [6] and found the time-varying gain. Then many researchers started investigating the problems of identification, analysis and control using different classes of OFs. The operational matrix for integration of BPFs was derived [8]. Moreover, it was shown that BPFs are more fundamental than WFs and the structure of the integration operational matrix of BPFs is simpler than that of WFs. In [9] it was shown that optimal control problems could be solved using BPFs with minimal computational effort.

In the last three and a half decades, the OF approach was successfully applied to study varieties of problems in systems and 
control $[22,57,59,61]$. The key feature of OFs is that it converts differential or integral equations into algebraic equations in the sense of least squares. So this approach became quite popular computationally as the dynamical equations of a system can be converted into a set of algebraic equations whose solution leads to the solution of the problem.

Before going into the details of the optimal control problem, we first take a look at the problem of estimation of state variables as the state estimation plays an important role in the context of state feedback control. State feedback control system design requires the knowledge of the state vector of the plant. Sometimes, no state variables or only a few state variables are available for measurement. In such cases an observer, either full order or reduced order depending on the situation, is incorporated to estimate the unknown state variables if the plant is observable. In general, it is estimated using the Luenberger observer [1]. But the Luenberger observer produces erroneous estimates in a noisy environment unless the measurement noise is filtered out. Interestingly, the OF approach has an inherent filtering property [59] as it involves an integration process which has the smoothing effect. As it appears from the literature, two attempts have been made on the state estimation problem by using two different classes of OFs, i.e. BPFs [23] and shifted Chebyshev polynomials of first kind (SCP1s)[39] so far. It is observed that the BPF approach [23] is purely recursive and uses multiple integration. The number of integrations increases as the order of the system increases, i.e for an $n^{\text {th }}$ order system the state equation has to be integrated $n$ times, which is computationally not attractive. 
Next, coming to the SCP1 approach [39], integration operational matrix of SCP1s is less sparse than that of shifted Legendre polynomials (SLPs). So if we use SLPs to develop algorithms, it will obviously be more elegant computationally. Moreover, state estimation cannot be done via SCP1s in a noisy environment.

The problem of optimal control incorporating observers has been successfully studied via different classes of OFs, namely BPFs [19], SLPs [30, 47], shifted Jacobi polynomials (SJPs) [33], general orthogonal polynomials (GOPs) [41], sine-cosine functions (SCFs) $[44,47]$, SCP1s [25, 47], shifted Chebyshev polynomials of the second kind (SCP2s) [47] and single-term Walsh series [52]. The approach followed in $[25,30,33,41,44,47]$ is nonrecursive, while it is recursive in $[19,52]$, making the approach in $[25,30,33,41,44,47]$ computationally not attractive.

Synthesis of optimal control laws for deterministic systems described by integro-differential equations has been investigated [11] via the dynamic programming approach. Subsequently, this problem has been studied via BPFs [37], SLPs [34, 43], and SCP1s [42].

The linear-quadratic-Gaussian (LQG) control problem [4] concerns linear systems disturbed by additive white Gaussian noise, incomplete state information and quadratic costs. The LQG controller is simply the combination of a linear-quadratic-estimator (LQE), i.e. Kalman filter, and an LQR. The separation principle guarantees that these can be designed and computed independently. In [35] the solution of the LQG control design problem has been obtained by employing GOPs. By using the GOPs the nonlinear Riccati differential equations have been reduced to nonlinear algebraic equations. The set of nonlinear algebraic equations has been solved to get the solutions. The above approach is 
neither simple nor elegant computationally, as nonlinear equations are involved.

Singular systems have been of considerable importance as they are often encountered in many areas. Singular systems arise naturally in describing large-scale systems [53]; examples occur in power and interconnected systems. In general, an interconnection of state variable subsystems is conveniently described as a singular system. The singular system is called generalized state-space system, implicit system, semi-state system, or descriptor system. Optimal control of singular systems has been discussed in [15] and [18]. In [38] the necessary conditions for the existence of optimal control have been derived. It has been shown that the optimal control design problem reduces to a two-point boundary-value (TPBV) problem for the determination of the optimal state trajectory. The single-term Walsh series method [56] has been applied to study the optimal control problem of singular systems. In [62] SLPs were used to solve the same problem. However, this approach is nonrecursive in nature. The Haar wavelet approach [68] has been presented to study the optimal control problem of linear singularly perturbed systems. In the recent times, SCFs [71], SCP1s [74] and Legendre wavelets [82] have been applied for solving the optimal control problem of singular systems. These approaches are again nonrecursive.

Time-delay systems are those systems in which time delays exist between the application of input or control to the system and its resulting effect on it. They arise either as a result of inherent delays in the components of the system or as a deliberate introduction of time delays into the system for control purposes. Examples of such systems are electronic systems, mechanical systems, biological 
systems, environmental systems, metallurgical systems, chemical systems, etc. A few practical examples [46] are controlling the speed of a steam engine running an electric power generator under varying load conditions, and control of room temperature, a cold rolling mill, spaceship, hydraulic system, etc.

As it appears from the literature, extensive work was done on the problem of optimal control of linear continuous-time dynamical systems containing time delays. Palanisamy and Rao [20] appear to be the first to study the optimal control problem via WFs. They considered time-invariant systems with one delay in state and one delay in control. In [24] time-varying systems containing one delay in state and one delay in control were considered, and optimal control problems of such systems was studied via BPFs. Solutions obtained in $[20,24]$ are piecewise constant. In order to obtain smooth solution, SCP1s [27] were used to study time-invariant systems with a delay in state only. In [31] time-varying systems with multiple delays in state and control have been studied via SLPs. The problem considered in [20] was investigated again by applying SLPs [36]. In [48] the problem investigated in [24] was again solved by approximating the time-delay terms via Pade approximation and using GOPs. Similarly, in [58] the problem considered in [27] was studied again via SLPs. Linear Legendre multiwavelets [81] were used to solve the time-varying systems having a delay in state only.

In recent years, people have come up with a new idea of defining hybrid functions (with BPFs and any class of polynomial functions ) and utilizing the same for studying problems in Systems and Control. The so-called hybrid functions approach was first introduced in [67] to study the optimal control problem of time- 
varying systems with a time-delay in state only. Subsequently, this approach was extended to time-invariant systems [72] studied in [27], delay systems containing reverse time terms [73], and timevarying systems [83] considered in [67]. In [84] general Legendre wavelets were used to solve the optimal control problem of timevarying singular systems having a delay in state only.

Looking at the historical developments on solving optimal control problem of nonlinear systems via the OF approach, we find that not much work has been reported. Lee and Chang [40] appear to be the first to study the optimal control problem of nonlinear systems using GOPs. For this, they introduced a nonlinear operational matrix of GOPs. Though their work is fundamental and significant, it is not attractive computationally, as it involves the Kronecker product [12] operation. Chebyshev polynomials of first kind (CP1s) were used [49] for solving nonlinear optimal control problems. In [54] a general framework for nonlinear optimal control problems was developed by employing BPFs.

A BPF approach to hierarchical control of linear time-varying large-scale systems has been reported [45]. The resulting solutions are found to be piecewise constant with minimal mean-squareerror.

\subsection{Organisation of the Book}

The book consists of eleven chapters in all. They are

Chapter 1: Introduction.

Chapter 2: Orthogonal Functions and Their Properties; in this chapter brief discussion on OFs and their classification are given. BPFs and their properties are given in Section 2.2. 
LPs are presented in Section 2.3. SLPs and their properties are discussed in Section 2.4. A nonlinear operational matrix using LPs and BPFs is introduced in Section 2.5.

Chapter 3: State Estimation; estimation of unknown state variables is done using full order observer, both in noisy and noise free environments. The algorithm in [23] is modified to deal with nonzero initial state vector.

Chapter 4: Linear Optimal Control Systems Incorporating Observers; using a reduced order observer the unknown state variables are estimated and the optimal control law is obtained.

Chapter 5: Optimal Control of Systems Described by IntegroDifferential Equations; a unique approach via BPFs or SLPs is presented in Section 5.2 to solve the optimal control problem of linear systems described by integro-differential equations.

Chapter 6: Linear-Quadratic-Gaussian Control; the LQG control design problem is discussed in Section 6.2. Solutions are given in Sections 6.3 and 6.4 which contain a unified approach and two recursive algorithms.

Chapter 7: Optimal Control of Singular Systems; in Section 7.2 a singular system problem is solved as an initial value problem under some conditions and two recursive algorithms are given. A more generalized solution to the problem is given in Section 7.3. 
Chapter 8: Optimal Control of Time-Delay Systems; an approach to compute optimal control law of linear time-varying multi-delay dynamic systems with a quadratic performance index is discussed in Section 8.2. Then application of the approach to

- Time-invariant systems

- Delay free systems

- Singular systems with delays

is given in Subsections 8.2.3, 8.2.4 and 8.2.5. In Section 8.3 optimal control of delay systems with reverse time terms is discussed and algorithms are presented.

Chapter 9: Optimal Control of Nonlinear Systems; a new method for computing optimal control law for nonlinear systems is given by employing LPs and BPFs.

Chapter 10: Hierarchical Control of Linear Systems; a BPF method of hierarchical control of linear time-invariant/timevarying large scale systems is presented.

Chapter 11: Epilogue; the last chapter concludes the book with the scope for further research. 


\section{References}

D. Luenberger , "Observing the state of a linear system," IEEE Trans. on Military Electronics, vol. 8, pp: 74-80, 1964.

J. J. Bongiorno, Jr. and D. C. Youla, "On observers in multivariable control systems," Int. J. Control, vol. 8, no. 3, pp: 221-243, 1968.

M. Athans and P. L. Falb , Optimal Control, Lincoln Laboratory Publications, 1968.

M. Athans, "The role and use of the stochastic linear-quadratic-Gaussian problem in control system design," IEEE Trans. Automatic Control, vol. 16, no. 6, pp: 529-552, 1971.

M. Kline, Mathematical thoughts from Ancient to Modern Times, Oxford University Press, New York, 1972.

C. F. Chen and C. H. Hsiao, "Walsh series analysis in optimal control," Int. J. Control, vol. 21, no. 6, pp: 881-897, 1975.

K. Ogata , State Space Analysis of Control Systems, Prentice-Hall Inc., Englewood Cliffs, N. J., 1976

C. F. Chen , Y. T. Tsay and T. T. Wu, "Walsh operational matrices for fractional calculus and their application to distributed systems," J. The Franklin Institute, vol. 303, no. 3, pp: 267-284, 1977.

P. Sannuti , "Analysis and synthesis of dynamic systems via block-pulse functions," Proc. IEE, vol. 124, no. 6, pp: 569-571, 1977.

A. P. Sage and C. C. White, Optimum Systems Control, Prentice-Hall, Inc., Englewood Cliffs, New Jersey, 1977. Y. A. Kochetkov and V. K. Tomshin, "Optimal control of deterministic systems described by integrodifferential equations," Automation and Remote Control, vol. 39, no. 1, pp: 1-6, 1978.

J. W. Brewer, "Kronecker products and matrix calculus in system theory," IEEE Trans. Circuits and Systems, vol. 25, no. 9, pp: 772-781, 1978.

M. G. Singh and A. Titli , Systems: Decomposition, Optimization and Control, Pergamon Press, Oxford, 1978.

G. X. Fang , Computational Methods of Optimal Control Problems, Beijing: Science Press, 1979.

L. Pandolfi, "On the regulator problem for linear degenerate control systems," J. Optimization Theory and Applications, vol. 33, no. 2, pp: 241-254, 1981.

N. S. Hsu and B. Cheng , "Analysis and optimal control of time-varying linear systems via block-pulse functions," Int. J. Control, vol. 33, no. 6, pp: 1107-1122, 1981.

M. L. Wang and R. Y. Chang, "Optimal control of linear distributed parameter systems by shifted Legendre polynomial functions," Trans. ASME J. Dynamic Systems, Measurement, and Control, vol. 105, no. 4, pp: 222-226, 1983.

D. Cobb , "Descriptor variable Systems and optimal state regulation," IEEE Trans. Automatic Control, vol. 28, no. 5, pp: 601-611, 1983.

S. Kawaji , "Block-pulse series analysis of linear systems incorporating observers," Int. J. Control, vol. 37, no. 5, pp: 1113-1120, 1983.

K. R. Palanisamy and G. P. Rao, "Optimal control of linear systems with delays in state and control via Walsh functions," Proc. IEE, pt. D, vol. 130, no. 6, pp: 300-312, 1983.

M. Jamshidi, Large-Scale Systems: Modelling and Control, North Holland, New York, 1983.

G. P. Rao, Piecewise Constant Orthogonal Functions and Their Application to Systems and Control, LNCIS 55, Springer, Berlin, 1983.

N. K. Sinha and Z. Q. Jie , "State estimation using block-pulse functions," Int. J. Systems Science, vol. 15, no. 4, pp: 341-350, 1984.

C. Hwang and Y. P. Shih , "Optimal control of delay systems via block-pulse functions," J. Optimization Theory and Application, vol. 45, no. 1, pp: 101-112, 1985.

J. H. Chou and I. R. Horng, "Shifted Chebyshev series analysis of linear optimal control systems incorporating observers," Int. J. Control, vol. 41, no. 1, pp: 129-134, 1985.

C. Hwang and M. Y. Chen, "Analysis and parameter identification of time-delay systems via shifted Legendre polynomials," Int. J. Control, vol. 41, no. 2, pp: 403-415, 1985.

I. R. Horng and J. H. Chou, "Analysis, parameter estimation and optimal control of time-delay systems via Chebyshev series," Int. J. Control, vol. 41, no. 5, pp: 1221-1234, 1985.

C. Hwang and M. Y. Chen, "Analysis and optimal control of time-varying linear systems via shifted Legendre polynomials," Int. J. Control, vol. 41, no. 5, pp: 1317-1330, 1985.

I. R. Horng and J. H. Chou, "Application of shifted Chebyshev series to the optimal control of linear distributed parameter systems," Int. J. Control, vol. 42, no. 1, pp: 233-241, 1985.

J. H. Chou and I. R. Horng, "Shifted Legendre series analysis of linear optimal control systems incorporating observers," Int. J. Systems Science, vol. 16, no. 7, pp: 863-867, 1985.

C. Hwang and M. Y. Chen, "Suboptimal control of linear time-varying multi-delay systems via shifted Legendre polynomials," Int. J. Systems Science, vol. 16, no. 12, pp: 1517-1537, 1985.

Y. F. Chang and T. T. Lee, "Applications of general orthogonal polynomials to the optimal control of time-varying linear systems," Int. J. Control, vol. 43, no. 4, pp: 1283-1304, 1986.

T. T. Lee, S. C. Tsay and I. R. Horng, "Shifted Jacobi series analysis of linear optimal control systems incorporating observers," J. The Franklin Institute, vol. 321, no. 5, pp: 289-298, 1986.

D. H. Shih and F. C. Kung, "Optimal control of deterministic systems via shifted Legendre polynonials," IEEE Trans. Automatic Control, vol. AC-31, no. 5, pp: 451-454, 1986. 
Y. F. Chang and T. T. Lee, "General orthogonal polynomials approximations of the linear-quadratic-Gaussian control design," Int. J. Control, vol. 43, no. 6, pp: 1879-1895, 1986.

M. H. Perng, "Direct approach for the optimal control of linear time-delay systems via shifted Legendre polynomials," Int. J. Control, vol. 43, no. 6, pp: 1897-1904, 1986.

C. Hwang , D. H. Shih and F. C. Kung, "Use of block-pulse functions in the optimal control of deterministic systems," Int. J. Control, vol. 44, no. 2, pp: 343-349, 1986.

V. Lovass-Nagy , R. Schilling and H. C. Yan, "A note on optimal control of generalized state-space (descripter) systems," Int. J. Control, vol. 44, no. 3, pp: 613-624, 1986.

J. H. Chou and I. R. Horng, "State estimation using continous orthogonal funtions," Int. J. Systems Science, vol. 17, no. 9, pp: 1261-1267, 1986.

T. T. Lee and Y. F. Chang, "Analysis, parameter estimation and optimal control of nonlinear systems via general orthogonal polynomials," Int. J. Control, vol. 44, no. 4, pp: 1089-1102, 1986.

Y. F. Chang and T. T. Lee, "General orthogonal polynomials analysis of linear optimal control systems incorporating observers," Int. J. Systems Science, vol. 17, no. 11, pp: 1521-1535, 1986.

D. H. Shih and L. F. Wang , "Optimal control of deterministic systems described by integrodifferential equations," Int. J. Control, vol. 44, no. 6, pp: 1737-1745, 1986.

J. H. Chou , "Application of Legendre series to the optimal control of integrodifferential equations," Int. J. Control, vol. 45, no. 1, pp: 269-277, 1987.

H. Y. Chung and Y. Y. Sun , "Fourier series analysis of linear optimal control systems incorporating observers," Int. J. Systems Science, vol. 18, no. 2, pp: 213-220, 1987.

J. M. Zhu and Y. Z. Lu , "New approach to hierarchical control via block-pulse function transformations," Int. J. Control, vol. 46, no. 2, pp: 441-453, 1987.

M. M. Zavarei and M. Jamshidi , Time-Delay Systems Analysis, Optimization and Applications, North-Holland Systems and Control Series, vol. 9, Amsterdam, 1987.

C. Y. Yang and C. K. Chen , "Linear optimal control systems by reduced-order observers via orthogonal functions," Int. J. Systems Science, vol. 19, no. 1, pp: 23-32, 1988.

S. C. Tsay , I. L. Wu and T. T. Lee, "Optimal control of linear time-delay systems via general orthogonal polynomials," Int. J. Systems Science, vol. 19, no. 2, pp: 365-376, 1988.

J. Vlassenbroeck and R. V. Dooren , "A Chebyshev technique for solving nonlinear optimal control problems," IEEE Trans. Automatic Control, vol. 33, no. 4, pp: 333-340, 1988.

J. M. Zhu and Y. Z. Lu , "Hierarchical optimal control for distributed parameter systems via block-pulse operator," Int. J. Control, vol. 48, no. 2, pp: 685-703, 1988.

J. M. Zhu and Y. Z. Lu, "Application of single step method of block-pulse functions to the optimal control of linear distributed parameter systems," Int. J. Systems Science, vol. 19, no. 12, pp: 2459-2472, 1988.

K. R. Palanisamy and K. G. Raghunathan, "Single-term Walsh series analysis of linear optimal control systems incorporating observers," Int. J. Systems Science, vol. 20, No. 7, pp: 1149-1155, 1989.

L. Dai , Singular Control Systems, Springer-Verlag, New York, 1989.

W. Shienyu , "Convergence of block pulse series approximation solution for optimal control problem," Int. J. Systems Science, vol. 21, no. 7, pp: 1355-1368, 1990.

M. Razzaghi and M. Razzaghi , "Fourier series approach for the solution of linear two-point boundary value problems with time-varying coefficients," Int. J. Systems Science, vol. 21, no. 9, pp: 1783-1794, 1990.

K. Balchandran and K. Murugesan, "Optimal control of singular systems via single-term Walsh series," Int. J. Computer Mathematics, vol. 43, no. 3, pp: 153-159, 1992.

Z. H. Jiang and W. Schaufelberger, Block-Pulse Functions and Their Applications in Control Systems, LNCIS 179, Springer, Berlin, 1992.

M. Razzaghi, M. F. Habibi and R. Fayzebakhsh, "Suboptimal control of linear delay systems via Legendre series," Kybernetica, vol. 31, no. 5, pp: 509-518, 1995.

K. B. Datta and B. M. Mohan, Orthogonal Functions in Systems and Control, Advanced Series in Electrical and Computer Engineering, vol. 9, World Scientific, Singapore, 1995.

M. Razzaghi and M. Habibi , "Application of Legendre series to the control problems governed by linear parabolic equations," Mathematics and Computers in Simulation, vol. 42, no. 1, pp: 77-84, 1996.

A. Patra and G. P. Rao, General Hybrid Orthogonal Functions and Their Applications in Systems and Control, LNCIS 213, Springer, London, 1996.

M. Razzaghi and M. Shafiee, "Optimal control of singular systems via Legendre series," Int. J. Computer Mathematics, vol. 70, no. 2, pp: 241-250, 1998.

S. A. Bianco , I. S. Sadek and M. T. Kambule, "Optimal control of a class of time-delayed distributed systems by orthogonal functions," J. The Franklin Institute, vol. 335, no. 8, pp: 1477-1492, 1998.

F. Fahroo and I. M. Ross, "Costate estimation by a Legendre pseudospectral method," J. Guidance, Control, and Dynamics, vol. 24, no. 2, pp: 270-277, 2001.

H. Jaddu, "Direct solution of nonlinear optimal control problems using quasilinearization and Chebyshev polynomials," J. The Franklin Institute, vol. 339, pp: 479-498, 2002.

H. R. Marzban and M. Razzaghi , "Hybrid functions approach for linearly constrained quadratic optimal problems," Applied Mathematical Modelling, vol. 27, no. 6, pp: 471-485, 2003.

H. R. Marzban and M. Razzaghi , "Optimal control of linear delay systems via hybrid of block-pulse and Legendre polynomials," J. The Franklin Institute, vol. 341, no. 3, pp: 279-293, 2004. 
H. R. Karimi , P. J. Maralani , B. Moshiri and B. Lohmann, "Numerically efficient approximations to the optimal control of linear singularly perturbed systems based on Haar wavelets," Int. J. Computer Mathematics, vol. 82, no. 4, pp: 495-507, 2005.

Y. Ordokhani and M. Razzaghi, "Linear quadratic optimal control problems with inequality constraints via rationalized Haar functions," Dynamics of Continous, Discrete and Impulsive Systems Series B: Applications and Algorithms, vol. 12, no. 5-6, pp: 761-773, 2005.

B. M. Mohan and S. K. Kar, "Comments on: Optimal control via Fourier series of operational matrix of integration", IEEE Transactions on Automatic Control, vol. 50, no. 9, 1466-1467, 2005.

R. Ebrahimi , M. Samavat, M. A. Vali and A. A. Gharavisi , "Application of Fourier series direct method to the optimal control of singular systems," ICGST-ACSE Journal, vol. 7, no. 2, pp: 19-24, 2007.

$X$. T. Wang, "Numerical solution of optimal control for time-delay systems by hybrid of block-pulse functions and Legendre polynomials," Applied Mathematics and Computation, vol. 184, no. 2, pp: 849-856, 2007.

X. T. Wang , "Numerical solution of optimal control for linear time-varying systems with delays via hybrid functions," J. The Franklin Institute, vol. 344, no. 7, pp: 941-953, 2007.

S. H. Chen, W. H. Ho and J. H. Chou, "Design of robust quadratic-optimal controllers for uncertain singular systems using orthogonal function approach and genetic algorithm," Optimal Control Applications and Methods, vol. 29, no. 5, pp: 373-391, 2008.

N. Boussiala , H. Chaabi and W. Liu , "Numerical methods for solving constrained nonlinear optimal control using the block pulse functions," Int. J. Innovative Computing, Information and Control, vol. 4, no. 7, pp: 1733-1740, 2008.

B. M. Mohan and S. K. Kar , "State estimation using shifted Legendre polynomials," Third Int. Conf. on Industrial and Information Systems (ICIIS), Kharagpur, INDIA, pp: 1-6, December 8-10, 2008.

B. M. Mohan and S. K. Kar, "Block-pulse functions approach to analysis of linear optimal control systems incorporating observers," Third Int. Conf. on Industrial and Information Systems (ICIIS), Kharagpur, INDIA, pp: 1-4, December 8-10, 2008.

B. M. Mohan and S. K. Kar, "State estimation using Block-Pulse Functions," IEEE INDICON, Kanpur, INDIA, pp: 280-285, December 11-13, 2008.

B. M. Mohan and S. K. Kar, "Shifted Legendre polynomials approach to analysis of linear optimal control systems incorporating observers," IEEE INDICON, Kanpur, INDIA, pp: 383-387, December 11-13, 2008.

S. K. Kar, "Orthogonal functions approach to optimal control of linear time-invariant systems described by integro-differential equations," KLEKTRIKA, vol. 11, no 1, pp: 15-18, 2009.

F. Khellat, "Optimal control of linear time-delayed systems by linear Legendre multiwavelets," J. Optimization Theory and Application, vol. 143, no. 1, pp: 107-121, 2009.

R. Ebrahimi , M. A. Vali , M. Samavat and A. A. Gharavisi, "A computational method for solving optimal control of singular systems using the Legendre wavelets," ICGST-ACSE Journal, vol. 9, no. 2, pp: 1-6, 2009.

M. Razzaghi , "Optimization of time delay systems by hybrid functions," Optimization and Engineering, vol. 10, no. 3, pp: 363-376, 2009.

$X$. T. Wang , "A numerical approach of optimal control for generalized delay systems by general Legendre wavelets," Int. J. Computer Mathematics, vol. 86, no. 4, pp: 743-752, 2009.

B. M. Mohan and S. K. Kar, "Optimal control of singular systems via block-pulse functions," Int. Conf. on Recent Advances in Mathematical Sciences and Applications, Gayatri Vidya Parishad, Visakhapatnam, INDIA, December 19-22, 2009.

B. M. Mohan and S. K. Kar, "Estimation and optimal control with reduced order observers via orthogonal functions," Int. J. Signal, System, Control and Engineering Application, vol. 3, no. 1, pp: 1-6, 2010.

B. M. Mohan and S. K. Kar, "Optimal control of multi-delay systems via orthogonal functions," Int. J. Advanced Research in Engineering and Technology, vol. 1, no. 1, pp: 1-24, 2010.

H. R. Marzban and M. Razzaghi , "Rationalized Haar approach for nonlinear constrained optimal control problems," Applied Mathematical Modelling, vol. 34, no. 1, pp: 174-183, 2010.

B. M. Mohan and S. K. Kar, "State estimation using orthogonal functions," Int. J. Mathematics and Engineering with Computers, vol. 1, no. 1-2, pp: 1-20, 2010.

B. M. Mohan and S. K. Kar, "Optimal control of multi-delay systems via block-pulse functions," Fifth Int. Conf. on Industrial and Information Systems (ICIIS), Mangalore, INDIA, pp: 614-619, July 29-August 1, 2010.

B. M. Mohan and S. K. Kar, "Orthogonal functions approach to optimal control of delay systems with reverse time terms," J. The Franklin Institute, vol. 347, no. 9, pp: 1723-1739, 2010.

B. M. Mohan and S. K. Kar, "Optimal control of singular systems via orthogonal functions," Int. J. Control, Automation and Systems, vol. 9, no. 1, pp: 145-152, 2011.

H. Ma , T. Qin and W. Zhang , "An efficient Chebyshev algorithm for the solution of optimal control problems,"

IEEE Trans. Automatic Control, vol. 56, no. 3, pp: 675-680, 2011.

B. M. Mohan and S. K. Kar, "Optimal control of multi-delay systems via shifted Legendre polynomials," Int. Conf. on Energy, Automation and Signals (ICEAS), Bhubaneswar, INDIA, December 28-30, 2011.

B. M. Mohan and S. K. Kar , "Optimal control of nonlinear systems via orthogonal functions," Int. Conf. on Energy, Automation and Signals (ICEAS), Bhubaneswar, INDIA, December 28-30, 2011. 
